\title{
STUDY OF VARIOUS MODIFICATIONS OF REVERSE SURAL ARTERY FLAP
}

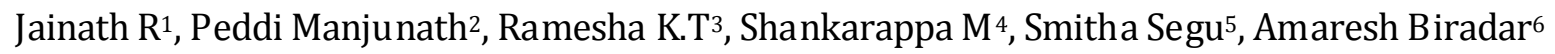

\section{HOW TO CITE THIS ARTICLE:}

Jainath R, Peddi Manjunath, Ramesha KT, Shankarappa M, Smitha Segu, Amaresh Biradar. "Study of various modifications of reverse sural artery flap". Journal of Evolution of Medical and Dental Sciences 2013; Vol. 2, Issue 44, November 04; Page: 8540-8546.

\begin{abstract}
Soft tissue reconstruction of distal third leg, heel and ankle region is a challenging problem because of poor vascularity and limited mobility of skin. The reverse sural artery with ideal flap thickness, minimal donor site morbidity, lack of functional muscle loss, short recovery time, wide arc of rotation and safe vascularity makes it a preferable flap for covering such defects. AIMS AND OBJECTIVES: To study various modifications of distally based reverse sural artery flap to suit the defects and for better survival of flaps. RESULTS: It is a retrospective study conducted in our institute to cover the distal leg and foot defects. 60 cases of distal leg defects exposing vital structure who underwent reverse sural artery flap coverage were included in the study .5 of the 60 flaps had complete flap necrosis and another 11 flaps had partial necrosis.73.4\% of the flaps survived during the follow up period of 1 years with good functional outcome. CONCLUSIONS: Reverse sural artery flap with its modifications is a good flap for the defects of distal leg, heel and ankle defects.
\end{abstract}

KEY WORDS: Reverse sural artery flap, modifications, distal leg defects.

Mesh terms: Reverse sural artery flap, modifications, distal leg defects.

INTRODUCTION: Soft tissue reconstruction of the lower third leg, ankle and foot region is a challenging problem. The major problem is the poor vascularity and limited mobility of the skin. Tendon, bone and hardware are frequently exposed because of the thinness of subcutaneous tissue making possibility of skin grafting a poor option. A durable flap with good skin texture, reliable vascularity, good arc of rotation, ease of dissection with minimum donor site morbidity is the most desired option for covering such defects.

The different local flaps for hind foot defects including dorsalis artery flap ${ }^{1}$, abductor hallucis and abductor digiti minimi muscle flaps ${ }^{2,3}$ have inadequate tissue and limited arc of rotation thereby limiting their frequent use. Medial plantar artery flap is an excellent option for weight bearing heel but its involvement in trauma frequently precludes its use.

Locoregional flaps for lower leg and ankle defects such as the peroneal artery flap, anterior tibial artery flap and posterior tibial artery flap ${ }^{4-6}$ have the disadvantage of sacrificing a major artery in already traumatized limb. Supramalleolar flap ${ }^{7}$ is another option but its reliability is questionable in compromised vascularity. Morbidity and operative times are increased in technically demanding microvascular surgeries ${ }^{8,9}$. Ideal flap thickness and quality, minimal donor site morbidity, the lack of functional loss, short recovery time , the wide arc of rotation and safe vascularity are the significant advantages of the reverse sural artery flap.

Masquelet et al ${ }^{10}$ Introduced the sural flap in 1992 with concise description of relevant anatomy and the surgical procedure. After the work of Masquelet et al distally based sural fasciocutaneous flap has become a mainstay in the reconstruction of the lower leg, ankle and heel defects. Over the past decade several modifications have been reported to improve flap viability and to solve a myriad of reconstructive needs. 
MATERIAL AND METHODS: A total of 60 cases of lower limb defects over lower third tibia and foot that were treated with distally based reverse sural artery flap in our institute over a period of 30 months from April 2011 to September 2013 constitute the material of this study. The various modifications of the flap included reverse sural island flap with a subcutaneous tunnel or externally placed pedicle, sural peninsular flap, sural fasciocutaneous flap, extended sural fascio musculocutaneous flap and delayed sural flap depending on the size and location of the defect. The defects included post traumatic wounds exposing vital structures or hardware and chronic non healing wounds requiring the flap cover. Chronic ulcers due to arterial and venous insufficiency were excluded from the study.

Relevant Anatomy: The sural artery arises from the popliteal artery and joins the sural nerve coursing between the two heads of gastrocnemius and follows the lateral edge of the Achilles tendon. The sural artery is intimately connected with the sural nerve and plays an important role in supplying the skin of lower and middle posterior leg. It terminates with the lateral supramalleolar branch of peroneal artery. Approximately four to eight perforators arise from the peroneal artery, pierce the crural fascia, and give rise to several branches that join adjacent perforators, forming an interconnecting vascular supra fascial plexus that extends from proximal part of leg to the posterior margin of lateral malleolus. A large perforator is located approximately $5 \mathrm{~cm}$ proximal to the lateral malleolus.

The lesser saphenous vein begins its course at the lateral border of the dorsum of foot, passes below the lateral malleolus, and travels along its posterior border. It then crosses the sural nerve after travelling approximately $1 / 3^{\text {rd }}$ of the length of the lower leg. More proximally, the lesser saphenous vein is located between the heads of the gastrocnemius muscle. The lesser saphenous vein remains superficial to the deep fascia until it passes through the popliteal fossa to drain into the popliteal vein.

In most cases the sural nerve is formed by the union of the medial and the lateral sural cutaneous nerve. The medial and lateral sural cutaneous nerves are branches of the tibial nerve and common fibular nerve, respectively.

\section{Surgical technique}

\section{Standard reverse sural island flap 11}

With patients in the prone position under general or spinal anesthesia the size of the defect is measured and a cutaneous island to be transferred is marked out on the middle or distal third of leg, depending on the length of pedicle necessary to reach the wound. The pedicle is kept centralised with regard to the flap. A line is marked from a point halfway between the Achilles tendon and the lateral malleolus extending to the midline between the two heads of gastrocnemius which roughly marks the course of the pedicle.

The pivot point is approximately $5 \mathrm{~cm}$ proximal to the lateral malleolus and posterior to the fibula. The axis of the pedicle is oblique and can be located precisely by the course of the lesser saphenous vein. A reliable adipofascial pedicle should not exceed the ratio of 4:1.

The incision begins over the proximal border of the skin island and is carried down to the sub fascial layer. The flap is elevated under tourniquet inflation. The sural nerve, and the accompanying short saphenous vein are identified, ligated and cut. The flap is dissected from 
proximal to distal, followed by the pedicle with an adjacent 1 to $2 \mathrm{~cm}$ width of fascia. The fascia must be included in both skin island and pedicle dissection. The viability of the flap is checked after tourniquet deflation. Hemostasis ensured. The flap is transferred with the pedicle which may be placed in a subcutaneous tunnel, open tunnel or externally placed with STG over it.

The secondary defect over donor site can be closed primarily when it is less than $4 \mathrm{~cm}$ in diameter. It should be covered with a partial thickness skin graft when it is larger.

\section{Various modifications of reverse sural artery flap}

1) Peninsular sural flap: Flap has a cutaneous artery and vein with overlying subcutaneous tissue and skin at its base. The inclusion of skin over the pedicle is a useful modification because it facilitates better manipulation of the flap and increases the margin of safety.

2) Sural artery fasciocutaneous flap ${ }^{12}$ : The inferiorly based fasciocutaneous flap with sural artery incorporation, which is perfused by peroneal perforators is an excellent modification and is more reliable. However disadvantage of the modification includes lesser arc of rotation and larger donor defect.

3) Sural fasciomusculocutaneous flap ${ }^{13}$ : The distally based sural fascio musculocutaneous flap, including a cuff of the gastrocnemius muscle along with sural nerve, can preserve the retrograde blood supply from the median superficial artery of the sural nerve to the musculocutaneous perforators of the gastrocnemius muscle over this critical area.

4) Delayed sural flap14: Experience with delay procedure has established the safety and reliability of larger flaps exceeding conventional flap dimensions in elderly patients with vascular risk factors. A delay period of 10 days seems to be appropriate in reconstruction of traumatized extre mities.

5) Supercharged reverse sural flap15: "Supercharging" means vascular augmentation of a flap by anastomosing the unrelated distant vascular source of flap, while "turbojetting" involves anastomosing the vascular source already present within the flaps pedicle to the flap.

6) Nerve sparing distally based sural fasciocutaneous flap 16 : Distally based sural nerve flap causes an area of parasthesia over the lateral aspect of foot. To prevent this sural nerve was dissected from the vein by microdissection and this did not compromise the vascularity of the flap with preservation of sensation.

RESULTS: Out of the 60 patients operated with reverse sural artery flap, 54 were male and 6 female patients with age group ranging from 3 to 70 years. The etiology of the defects included posttraumatic, post infective, post burn non healing ulcers and pressure ulcers over heel. 22 of the defects were around the medial malleoli, 16 over the heel, 11 over anterior ankle and foot, 9 over the lower $1 / 3^{\text {rd }}$ leg and 2 over lateral malleoli. There was an underlying bone, fracture , implant or tendon exposed in all patients. 43 of this patients underwent standard reverse sural island flap,10 reverse sural fasciocutaneous flap, 3 delayed sural flap, 2 sural fascio musculocutaneous flap and 2 peninsular sural flap .The size of the defects for island flaps ranged from $5 \times 5 \mathrm{~cm}$ to $9 \times 8 \mathrm{~cm}$. The 


\section{ORIGINAL ARTICLE}

length of sural fasciocutaneous flaps ranged from 7 to $25 \mathrm{~cm}$ from the tip of lateral malleolus. 5 of these flaps underwent complete flap necrosis and 11 had partial necrosis which was later covered with skin grafting after granulation.
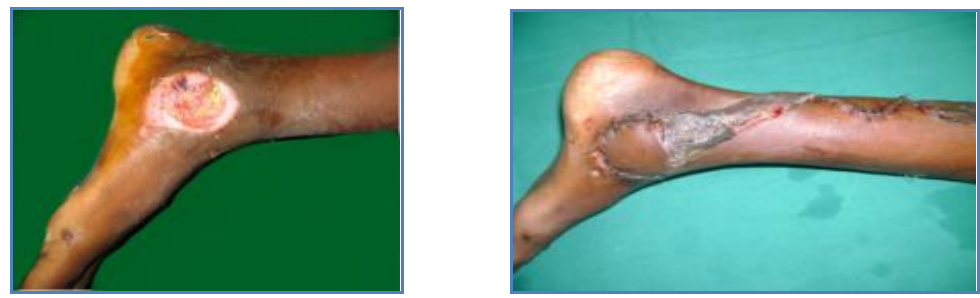

Fig. 1: Reverse sural island flap with externally placed pedicle.
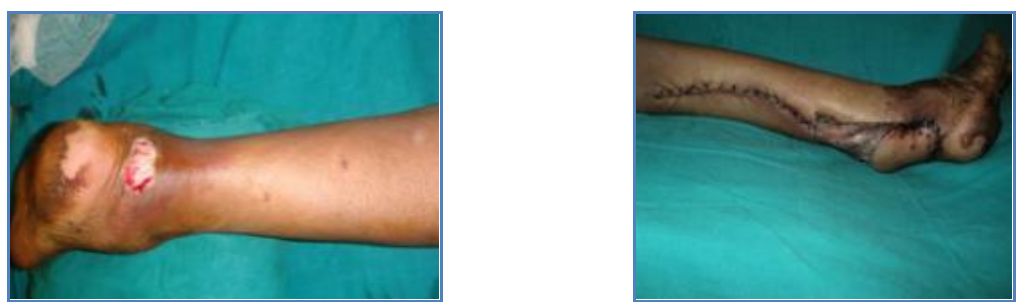

Fig. 2: Reverse sural island flap. Secondary defect primarily closed.
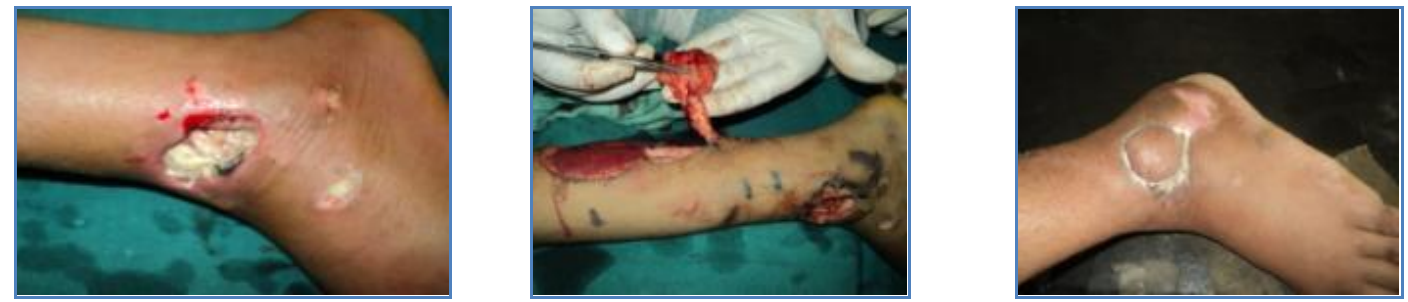

Fig. 3: Sural island flap with subcutaneous tunneling of pedicle
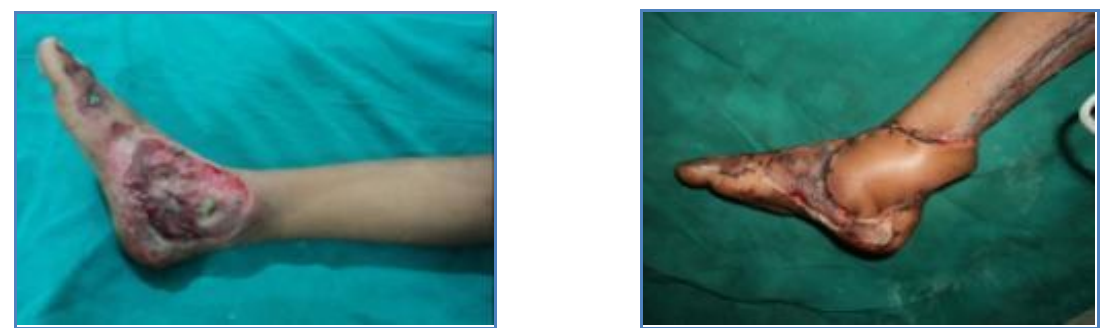

Fig. 4: Sural artery peninsular flap 


\section{ORIGINAL ARTICLE}

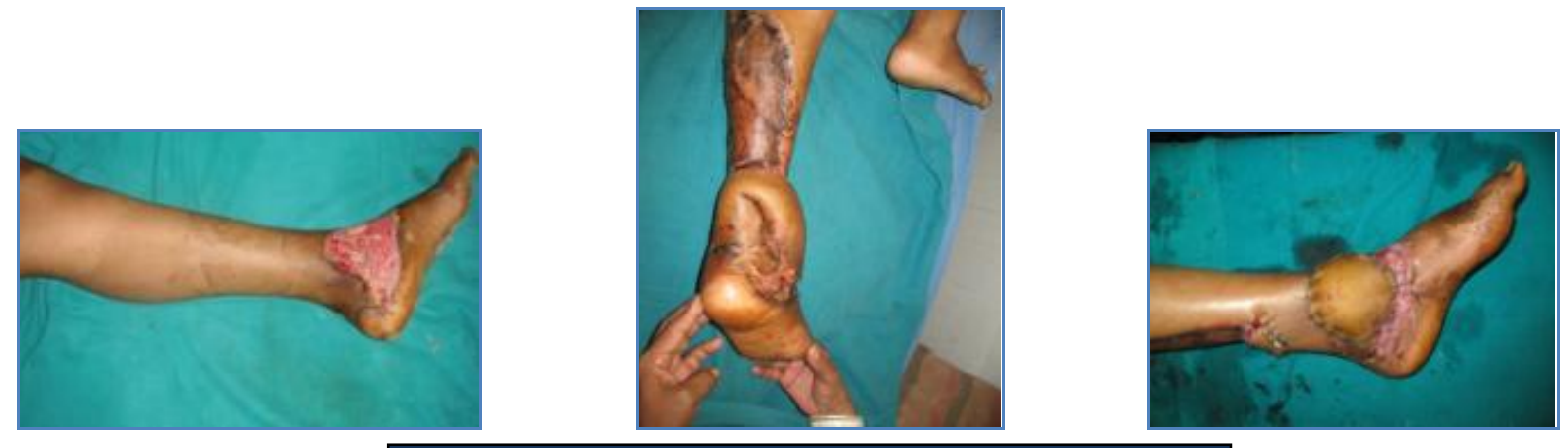

Fig. 5: Reverse sural artery fasciocutaneous flap.
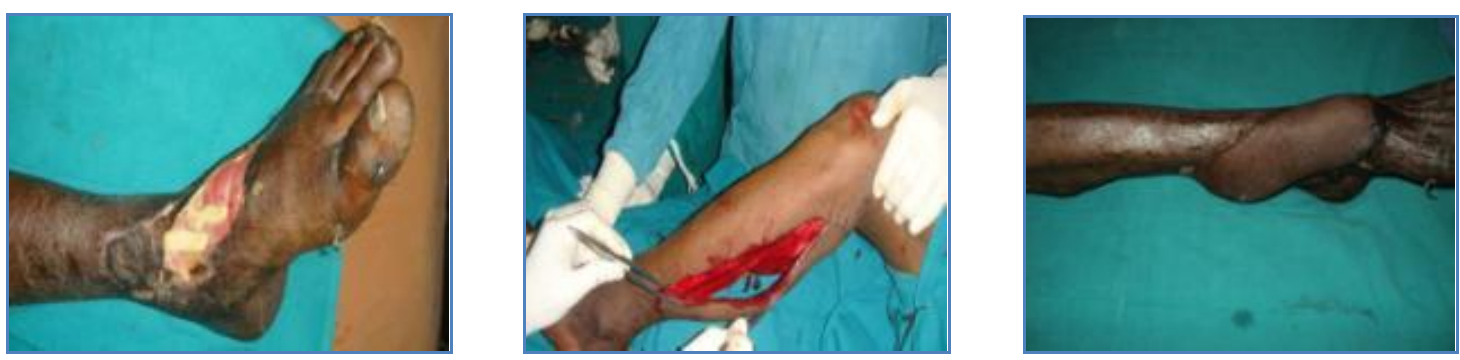

Fig. 6: Delayed reverse sural artery flap.
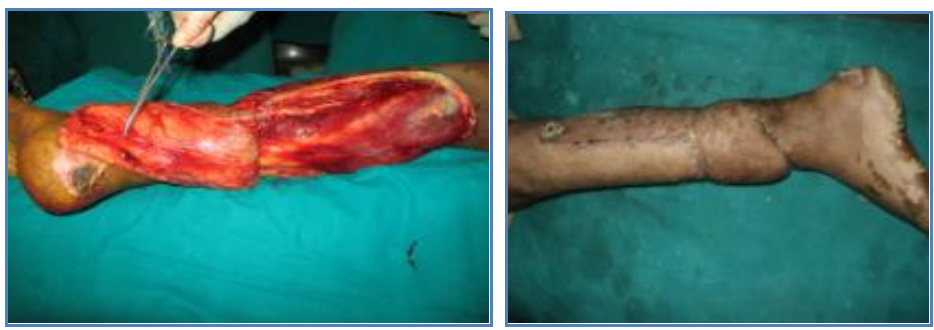

Fig. 7: Extended sural fascio musculocutaneous flap.

DISCUSSION: Reverse sural artery flap remains the workhorse flap for reconstruction of foot and ankle defects. The constant and reliable blood supply with ease of elevation makes it a preferable flap for these defects. The various modifications of the flap are done to suit clinical conditions and for better survival of the flap. Distal sural artery flap can be used to cover defects over medial and lateral malleoli, over dorsum of foot and ankle and weight bearing heel. Flap is safe in paediatric age group, with no difference in complication rates. Inclusion of both the accompanying vascular territories of the sural nerve as the main arterial supply and the lesser saphenous vein as an additional blood supply and to augment the venous drainage of the flap improves survival. Inclusion of skin over the pedicle as in peninsular and fasciocutaneous flaps facilitates better manipulation of the flap and increases margin of safety. Flap can be safely elevated from proximal $1 / 3^{\text {rd }}$ of leg by inclusion of mesentery like tissue between gastrocnemius muscles or a chunk of gastrocnemius muscle itself. Delay flap is preferred in high risk patients with diabetes, smoking and hypertension. The long pedicle sural artery fasciocutaneous flap is preferred in patients who have large defects 
over the weight bearing heel and over foot. Large donor site deformity was the disadvantage with long pedicle fasciocutaneous flap but with the advantage of better survival. Flap edema and venous congestion were the most common complications in immediate post operative period, which settled with no serious problems. Loss of sensation over the lateral aspect of foot was a common but not a major complaint in late follow up.

CONCLUSSION: The distally based reverse artery flap with its various modifications is an ideal flap for covering defects over distal $1 / 3$ rd leg, ankle and proximal foot defects.

\section{REFERENCES:}

1. Ishikawa K, Isshiki N, Suzuki S, et al. Distally based dorsalis pedis island flap for coverage of the distal portion of the foot. Br J Plast Sur 1987; 40:521-5.

2. Mathes SJ, Nahai F. Abductor hallucis muscle flap. Clinical atlas of muscle and musculocutaneous flaps. St Louis: CV Mosby; 1979 p. 269-277.

3. Yoshimura Y, Nakajima T, Kami T. Distally based abductor digiti minimi muscle flap. Ann Plast Surg 1985; 14:375-7.

4. Wee JT. Reconstruction of the lower leg and foot with the reverse-pedicled anterior tibial flap: preliminary report of a new fasciocutaneous flap. Br J Plast Surg 1986; 39:327-37.

5. Liu K, Li Z, Lin Y, et al. The reverse-flow posterior tibial island flap: anatomic study and 72 clinical cases. Plast Reconstr Surg 1990; 86:312-6.

6. Yoshimura M, Imura S, Shimamura K, et al. Peroneal flap for reconstruction in the extremity: preliminary report. Plast Reconstr Surg 1984; 74:402-9.

7. Masquelet A, Beveridge J, Romana C, Gerber C. The lateral supramalleolar flap. Plast Reconstr Surg 1988; 81: 74-81.

8. Swartz WM, Mears DC. The role of free-tissue transfers in lower extremity reconstruction. Plast Reconstr Surg 1985; 76:364-73.

9. Stevenson TR, Mathes SJ. Management of foot injuries with free-muscle flaps. Plast Reconstr Surg 1986; 78:665-71.

10. Masquelet AC, Romana MC, Wolf G. Skin island flaps supplied by the vascular axis of the sensitive superficial nerves: anatomic study and clinical experience in the leg. Plast Reconstr Surg 1992; 89:1115-21.

11. Al Qattan MM: A modified technique for harvesting the reverse sural artery flap from the upper part of the leg: inclusion of gastrocnemius muscle 'cuff' around the sural pedicle. Ann Plast Surg 2001, 47(3):269 274.

12. Price MF, Capizzi PJ, Watterson PA, Lettieri S (2002) Reverse sural artery flap: caveats for success. Ann Plast Surg 48:496-504

13. Shao-Liang Chen, Tim-Mo chen, Hsian-Jenn Wang; The distally based sural fascio musculocutaneous flap for foot reconstruction. J of Plast Reconst Aesth Surg: 2006, 59,846855.

14. Ulrich Kneser, M.D., Alexander D. Bach, Delayed reverse sural flap for staged reconstruction of the foot and lower leg. Plast. Recons Surg .116:7, 2004.

15. Onder Tan, M.D., Bekir Atik, M.D and Mehmet: Supercharged reverse flow sural flap; A new modification increasing the reliability of the flap. Microsurgery 25; 36-43, 2005. 
16. Osman E Aydin, Onder Tan, Selma et al ;Nerve sparing distally based sural flap. Microsurgery 31, 4,276-280; 2011.

\section{AUTHORS:}

1. Jainath R.

2. Peddi Manjunath

3. Ramesha K.T.

4. Shankarappa M.

5. Smitha Segu

6. Amaresh Biradar

\section{PARTI CULARS OF CONTRIBU TORS:}

1. Assistant Professor, Department of Plastic Surgery, Victoria Hospital, BMCRI.

2. Assistant Professor, Department of Plastic Surgery, Victoria Hospital, BMCRI.

3. Associate Professor, Department of Plastic Surgery, Victoria Hospital, BMCRI.

4. Professor, Department of Plastic Surgery, Victoria Hospi tal, BMCRI.
5. Associate Professor, Department of Plastic Surgery, Victoria Hospital, BMCRI.

6. Post Graduate, Departm ent of Plas tic Surgery, Victoria Hospi tal, BMCRI.

\section{NAME ADDRESS EMAIL ID OF THE CORRESPONDING AUTHOR:}

Dr. Jainath $\mathrm{R}$, No. $3,14^{\text {th }}$ Cross, Vasanth Nagar, Bangalore - 560052 .

Email -drjainathr@gmail.com

Date of Submission: 03/10/2013. Date of Peer Review: 04/10/2013. Date of Acceptance: 10/10/2013. Date of Publishing: 29/10/2013 